Cahiers d'études italiennes

12 | 2011

Texte et image dans la culture italienne

\title{
Deviazioni dalla norma prospettica nella pittura del XV secolo. Soluzioni eterodosse nel san Sebastiano di Antonello da Messina
}

Alessia Pipitone

\section{OpenEdition}

\section{Journals}

Edizione digitale

URL: http://journals.openedition.org/cei/596

DOI: $10.4000 /$ cei.596

ISSN: 2260-779X

\section{Editore}

UGA Éditions/Université Grenoble Alpes

\section{Edizione cartacea}

Data di pubblicazione: 15 mars 2011

Paginazione: 127-144

ISBN: 978-2-84310-190-8

ISSN: 1770-9571

\section{Notizia bibliografica digitale}

Alessia Pipitone, «Deviazioni dalla norma prospettica nella pittura del xv secolo. Soluzioni eterodosse nel san Sebastiano di Antonello da Messina», Cahiers d'études italiennes [Online], 12 | 2011, online dal 15 septembre 2012, consultato il 26 mars 2021. URL: http://journals.openedition.org/cei/596 ; DOI: https://doi.org/10.4000/cei.596 


\title{
DEVIAZIONI DALLA NORMA PROSPETTICA NELLA PITTURA DEL XV SECOLO. SOLUZIONI ETERODOSSE NEL SAN SEBASTIANO DI ANTONELLO DA MESSINA
}

\author{
Alessia Pipitone \\ Roma
}

Nella tavola del san Sebastiano della Gemäldegalerie di Dresda ${ }^{\mathrm{I}}$, che i recenti restauri hanno rivelato e riportato all'attenzione del grande pubblico, Antonello da Messina, autore del dipinto mostra la sua abilità prospettica; la prospettiva quattrocentesca, la scienza sulla quale si misurano tutti i pittori dell'epoca, non è però da intendersi come norma assoluta ma strumento nelle mani dell'artista. Se questo è vero allora si può ipotizzare che proprio là dove gli impianti prospettici dei grandi maestri presentano soluzioni eterodosse rispetto alla norma codificata, si possano coagulare i più interessanti dubbi e interrogativi circa le ragioni, gli effetti e i significati della prospettiva stessa.

Nell'ambientazione del san Sebastiano le proporzioni imposte dalla regola prospettica sembrano trascurate in favore di una rappresentazione monumentale e drammatica della figura principale; le irregolarità nell'organizzazione della luce e delle ombre, coerenti con la deformazione prospettica, sembrano affermare la volontà di accrescere l'effetto espressivo dell'opera e di sollecitare una fruizione partecipata.

La deroga dalla norma si presenta più come una ricerca fortemente significante che come un errore d'applicazione e permette di formulare delle ipotesi intorno alle modalità di fruizione promosse e imposte dall'opera.

I. L'opera, originariamente su tavola, è stata trasferita su tela in una data imprecisata ma comunque prima dell'acquisizione della stessa da parte della Pinacoteca di Dresda. 


\section{La tavola}

Complice un orizzonte molto ribassato, il san Sebastiano, legato a un improbabile tronco d'albero, domina su tutta la scena; è leggermente inclinato, poggiato al tronco, con gli occhi rivolti verso 'alto $^{2}$. Alle sue spalle una fuga vertiginosa e molto scorciata mostra una strana piazza pavimentata a mattonelle; sembra di vedere dell'acqua oltre i volumi cristallini dei portici, oltre la sospensione delle figure in lontananza (si veda fig. I).

Antonello attualizza episodio e scena, ripropone il martirio del santo in una giornata qualunque, in una piazza del suo tempo perfettamente credibile e unitaria, di uno straordinario naturalismo debitore forse dei fiamminghi nell'attenzione ai dettagli di vita quotidiana. Tale concretezza si scontra però con l'inverosimile albero che esce dalla pavimentazione in prospettiva e con l'assoluta normalità delle faccende da cui sono presi i vari personaggi della scena come non vedessero l'uomo legato e trafitto. Qualcosa sfugge a un'immediata comprensione e accresce il potere di fascinazione di quest'opera. Qualche cosa d'incerto e forse non coerente sollecita l'attenzione: qualche errore nell'impianto prospettico, qualche cosa che non torna nella costruzione delle ombre, un sapore noto nell'inaccessibile sospensione dei personaggi. Ma queste discordanze non costituiscono forse «[...] la molla della ricerca del senso, l'imperfezione irriducibile che impedisce di ridurre l'emozione estetica ad una semplice percezione o ad un gioco di inferenze cognitive?»3.

E un invito a scandagliare ancora, centimetro per centimetro, l'opera, «l'osservatore è condotto a riconoscere nell'insoddisfazione un'intenzionalità del visibile» ${ }^{4}$.

2. Una grande tavola dipinta ad olio raffigurante un san Sebastiano è menzionata per la prima volta dal Sansovino sull'altare della confraternita di san Rocco nella chiesa veneziana di san Zulian; la pala d'altare era composta da un San Rocco, poi danneggiato dal crollo di una parte del soffitto e sostituito da una statua lignea, da un San Cristoforo di Antonello e dal San Sebastiano. Studi recenti hanno fatto ipotizzare una datazione piuttosto avanzata della tavola, che risalirebbe all'ultimo periodo messinese, probabilmente il I478, cfr. M. Lucco (ed.), Antonello da Messina, Milano, Silvana Editore, 2006.

3. J. Fontanille, "Senza titolo o senza contenuto?», in Leggere l'opera d'arte II, Bologna, Esculapio, I999, p. 65 .

4. Ibid., p. 66. 


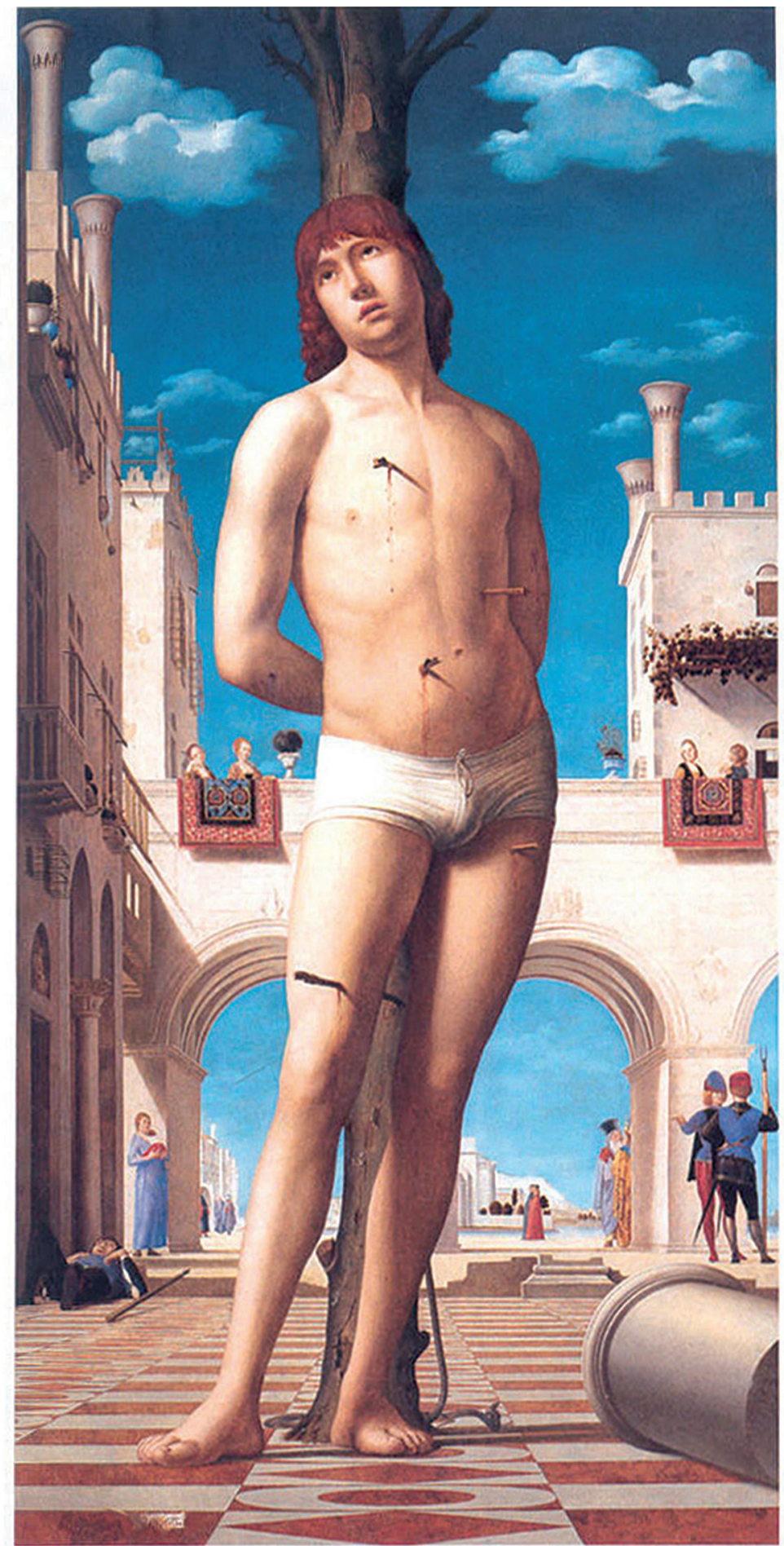

Fig. I, Antonello da Messina, San Sebastiano, Dresden Gemäldegalerie. 


\section{Soluzioni prospettiche devianti}

Nell'applicazione rigorosa e scientifica della norma prospettica codificata, Antonello sembra essersi concesso una serie di licenze e tali deroghe sono troppo studiate perché siano considerate errori, forse sono, invece, funzionali a un'esigenza espressiva. Le licenze prospettiche indicano un'attenzione alla posizione dell'osservatore e si coagulano intorno a san Sebastiano; ne risulta dunque ridefinita la posizione dell'osservatore rispetto alla figura del santo - a quel corpo sacro oggetto di preghiera e venerazione - rimandando a quella straordinaria rivoluzione compiuta in questo senso da Mantegna con il Cristo in scurto.

La prospettiva è un capitolo nuovo nella storia dell'arte, una forma nuova in grado di tradurre quella particolare visione del mondo che è del Quattrocento, «una forma simbolica», dice Panofsky', l'espressione sensibile di quella cultura. I modelli di funzionamento della cultura quattrocentesca, a tutti i livelli, tecnici, materiali, scientifici, filosofici, fino al quotidiano di ogni individuo, troverebbero una loro estrinsecazione pittorica nell'invenzione della prospettiva.

La creazione di uno spazio credibile, che già dalle ricerche empiriche di Giotto avevamo visto accompagnata a un'umanizzazione delle figure sacre, acquista ora una pregnanza maggiore: tutto quanto viene inserito in questo spazio misurabile diviene oggetto di conoscenza, così sarà per i santi o per Cristo che acquistano ora un aspetto inedito, umano, adatto anche a una nuova forma di devozione. Già Longhi e poi Zeri, coniando le etichette di Rinascimento umbratile il primo e di Pseudo-Rinascimento il secondo, hanno posto l'accento sul sistema prospettico come sistema complessivo di guardare e rappresentare il mondo:

Esso [lo stile rinascimentale] nacque quando le ricerche spaziali (cioè di rappresentazione tridimensionale) già presenti in Giotto e nei suoi seguaci (specie Taddeo Gaddi e Maso di Banco) passarono dalla sperimentazione intuitiva e personale alla sistematizzazione scientifica, grazie a Filippo Brunelleschi. Corollario essenziale di tale ricerca dello spazio figurativo è lo studio del corpo umano nella sua struttura interna, dell'espressione dei sentimenti e dei moti psicologici in quanto riflessi di un autentico stato d'animo e non di norme dettate da mode o da abitudini di comportamento sociale. Altro corollario di tale situazione mentale è l'assenza di ornamenti pletorici, di calligrafismi, di cifre esornative; [...] È ovvio che uno stile caratterizzato da connotati siffatti, non coincide con il Rinascimento inteso come periodo storico; al

5. E. Panofsky, La prospettiva come forma simbolica, Milano, Feltrinelli, I99I. 
contrario esso fu assai limitato svolgendosi tra le continue riprese di modi e di stilemi formali ad esso opposti, e che spesso ne deviarono l'essenza e l'intimo significato ${ }^{6}$.

La nascita della scienza prospettica accelera il processo di emancipazione dell'artista dalla categoria degli artigiani per avvicinarlo agli intellettuali; misurare è un modo per conoscere e l'arte diviene strumento di conoscenza $^{7}$. Poter finalmente dedurre dalla percezione la reale dimensione e collocazione delle cose è una grande conquista per un uomo che tenta di imporre il suo dominio sul mondo in una progressiva acquisizione di consapevolezza delle sue capacità. È un modo diverso di apprendere, prima ancora che di rappresentare la realtà; ciò che è possibile conoscere è possibile possedere. L'artista partecipa di questo e risponde a nuove esigenze, frutto di un pensiero rinnovato che va sotto il nome di Umanesimo. Poter scegliere a proprio piacimento dove collocare l'osservatore, e da questo far discendere, rigorosamente, tutto lo spazio circostante, afferma e ci informa circa la centralità dell'uomo in questo sistema culturale.

Si legge ovunque del rigore scientifico degli artisti del Quattrocento nell'applicare la prospettiva, quasi fosse virtuosismo fine a se stesso, quasi che la nuova scienza togliesse in libertà dando in potenzialità, ma l'espressione artistica usa e piega gli strumenti ai suoi fini e la conoscenza del mondo che la prospettiva porta con sé non è il solo metro di misura di un'opera d'arte.

\section{La restituzione prospettica}

Il san Sebastiano si trova in un'ampia piazza che termina con un portico oltre il quale s'intravede dell'acqua e forse un'isola della laguna veneta. La pavimentazione a mattonelle alternate fornisce, oltre a chiarissime linee di fuga, la diagonale del quadrato, unico strumento noto, e suggerito dall'Alberti, per individuare il punto di distanza. Questo si trova collocato fuori del quadro, lontano. Il punto di fuga, e dunque il punto di vista, è posto perfettamente al centro del lato corto della tavola ma molto in basso, in corrispondenza del polpaccio sinistro del santo ${ }^{8}$.

Riportando le dimensioni delle figure in secondo piano fino alla linea di terra posta in corrispondenza del margine inferiore del quadro, attraverso le linee di fuga, si nota subito una forte discrepanza con la teoria

6. F. Zeri, «Rinascimento e Pseudo-Rinascimento», in Storia dell’arte italiana, vol. I, parte II, Torino, Einaudi, I978, p. 547 .

7. Marsilio Ficino suggeriva agli artisti di sviluppare le facoltà mercuriali: contare, pesare, misurare.

8. Le riflettografie ad infrarossi effettuate in occasione dei recenti restauri hanno mostrato il reticolo prospettico tracciato da Antonello attraverso incisioni realizzare probabilmente con una punta metallica. 
prospettica che vorrebbe le cose e le persone dimensionate in relazione al posto da loro occupato nello spazio: san Sebastiano è notevolmente più alto pur trovandosi su di un piano arretrato di qualche mattonella rispetto alla linea di terra.

La perfetta costruzione prospettica di ogni parte dell'opera allontana il sospetto di un involontario errore del maestro nel dimensionamento della figura di Sebastiano, sarebbe questo l'unico errore, difficile credere che Antonello si sia sbagliato proprio nella realizzazione della figura principale.

Riportando, in un'ipotetica ricostruzione della piazza, il portico di fondo fino alla linea di terra alla destra di Sebastiano ci si accorge dell'improbabile altezza di quest'ultimo che va a superare di molto la linea d'imposta dell'arco, cioè quella linea immaginaria da cui si alza l'arco al di sopra dei pilastri, mentre ogni altra parte del dipinto si organizza su rapporti metrici credibili. Va inoltre osservato che la figura, anche presa al di fuori del suo contesto, non subisce alcuna riduzione metrica delle sue parti. In una visione prospettica dal basso i rapporti proporzionali mutano, il corpo risulta ridotto nelle parti più alte, dunque più lontane dall'osservatore, le proporzioni si alterano tanto più l'osservatore si trova in basso rispetto alla figura. In questo caso, invece, il san Sebastiano si presenta in tutta la sua altezza; da una parte il sovradimensionamento prospettico lo presenta maestoso, dall'altra i suoi stessi rapporti interni, per niente ridotti, risultano impostati su una ripetizione modulare piuttosto alta: 8,3 volte la testa ${ }^{9}$. Il corpo del santo risulta dunque non subire le riduzioni che la prospettiva richiede. Se pur inserito in un ambiente credibile e coerentemente organizzato, Sebastiano non sottostà a queste leggi.

Antonello sembra giocare dunque la sua opera su più piani di lettura. Il martire è, prima di tutto, un uomo il cui corpo ferito si mostra nella sua assoluta consistenza umana, di carne, di sangue che esce dalle ferite delle frecce, ma le sue dimensioni incoerenti attestano il suo essere fatto di una materia spirituale: ferito nel corpo come un uomo ma libero dai vincoli che lo spazio dell'uomo impone ai corpi. Ciò che vale per le figure presenti alla scena, per gli stessi arcieri che hanno scagliato le frecce, per coloro che si trovano a disporre della vita del martire, non vale per lui che pure di quelle frecce sembra stia morendo, ferito nel corpo attesta la sua libertà dalle leggi del suo stesso corpo, difficile immaginare una più efficace definizione di martirio.

9. I vari canoni per la rappresentazione della figura umana riportano una modularità di circa 7 o 8 volte la testa. Va inoltre osservato che il rapporto indicato $(8,3)$ è arrotondato per difetto, essendo calcolato su di un corpo inclinato e ruotato. Se immaginiamo che il Santo si raddrizzi dalla sua posizione il rapporto aumenterebbe e il torace diverrebbe ancora più lungo contraddicendo, ancor più, ogni logica prospettica. 


\section{La luce e l'ombra}

Attraverso luce e chiaroscuro l'artista modella i corpi e le cose; le ombre rendono credibile lo spazio e tridimensionali le figure, riportano gli oggetti a una concretezza percettiva. Nel Quattrocento la luce ha acquistato ormai una sua autonomia dal solo referenzialismo simbolico, è strumento del pittore che vuole offrire un'immagine di verità ottica; senza perdere necessariamente la sua valenza simbolica, è ora oggetto di un'attenzione di tipo naturalistico.

$\grave{E}$ noto il ruolo avuto dalle esperienze fiamminghe nella conquista di una consapevolezza nei confronti delle potenzialità della luce da parte di Antonello e, più in generale e attraverso suo tramite, gli effetti sul panorama figurativo della penisola.

Incerta la cronologia degli spostamenti di Antonello cui Vasari attribuisce un fondamentale - ma assolutamente improbabile - viaggio nelle Fiandre. È certo, invece, che la sua formazione avviene anche su testi figurativi fiamminghi, borgognoni e catalani presenti nel sud d'Italia, formazione che resterà tratto distintivo della sua poetica rendendolo particolarmente sensibile alle problematiche della luce e portandolo a quella «miracolosa sintesi di resa fiamminga d'atmosfera e di visione prospettica italiana ${ }^{\text {10 }}$. Ma nella sintesi antonelliana emergono evidenti le specificità e le distanze rispetto alle soluzioni fiamminghe:

In Antonello è anzitutto una luminosità silente e permeante, meticolosamente misurata con l'ombra, che grazie a un'abilissima sintassi prospettica concorre a rendere ogni cosa stereometrica, tersa e distinta. Altro dunque dalla luce fiamminga, meno incline al valore plastico - e classico - e più a quel potenziamento luminoso delle superfici pittoriche che svela del reale la qualità particolarissima, la texture inconfondibile ${ }^{\mathrm{II}}$.

E si può dire, infatti, che

[...] la robusta capacità di sintesi spaziale dell'artista messinese, sottostante a quella pelle prodigiosamente mimetica, sembra aver rivelato alla committenza veneziana l'intera gracilità stilistica dei dipinti framminghi, determinandone un rapido tramonto nel gusto $[\ldots]^{12}$.

\footnotetext{
IO. M. Lucco, «Venezia» in La pittura nel Veneto. Il quattrocento, Milano, Electa, I990, t. II, p. 444.

II. G. Poldi, G. Villa, «Cuius pictura est intuenda admirationi. Contributo alla comprensione della tecnica di Antonello", in Antonello da Messina, l'opera completa, M. Lucco (ed.), Milano, Silvana editore, 2006, p. 9I e seg.

I2. M. Lucco, op. cit. p. 445
} 
Alba o tramonto, quale possibile interpretazione per una luce così ambigua?

La luce di Antonello assolve la medesima funzione della prospettiva, la lezione fiamminga suggeriva la possibilità di trovare quell'unitarietà spaziale - che in Italia era raggiunta con gli strumenti prospettici - attraverso la luce; una luce determinata, che potesse scivolare sugli oggetti e sui corpi rivelandone ogni valore tattile. Stoffe, tessuti, gemme, vetri, incarnati vengono esibiti nel loro valore epidermico oltre che plastico; una sensibilità tanto diversa da quella italiana che Antonello sa recepire e sintetizzare superando l'atteggiamento analitico della concezione fiamminga.

Nel san Sebastiano di Dresda la luce proietta ombre lunghe, modella il corpo del santo e sottolinea il rilievo delle frecce, ma malgrado quest'alta definizione luministica, è difficile stabilire l'ora del giorno; Leonardo Sciascia vi vedeva un assolato pomeriggio messinese ${ }^{13}$, ma le ombre in parte lo contraddicono.

Attraverso il proiettarsi delle ombre del corpo di san Sebastiano, con un certo margine d'approssimazione, si può rintracciare la fonte di luce collocata alle spalle dell'osservatore e piuttosto in basso. Sebastiano è dunque illuminato da un sole basso, ma la traiettoria dell'ombra del suo corpo differisce notevolmente da quella degli edifici sullo sfondo. Gli elementi architettonici rispondono, infatti, a una fonte di luce ben diversa, posta, approssimativamente, in alto alla destra del santo. Se l'intera piazza fosse stata organizzata secondo la medesima luce che illumina il corpo di Sebastiano, si vedrebbero ombre lunghe coprire buona parte del pavimento della piazza e il rocchio di colonna sarebbe illuminato nella sua parte visibile proiettando un'ombra alla nostra destra, fuori quadro. Antonello ha scelto, dunque, diversi punti di luce, uno per il santo e uno per le architetture, differenziando il martire dal resto e attribuendogli una luce, dunque una posizione del sole (alba o tramonto?), leggibile in chiave simbolica.

La luce unifica a livello percettivo, rende concreta e tangibile l'immagine, scolpisce il corpo e lo rende umano, Sebastiano è una figura di carne che proietta un'ombra come ogni altro corpo, poggia con i piedi su un reale pavimento, è concreto, è un uomo, ma per lui Antonello immagina un sole diverso; un'altra sottile ambiguità che contraddice la dimensione tutta concreta della scienza prospettica. 


\section{Il problema dello scorcio}

La visione dal basso che Antonello ha adottato per quest'opera richiama alla mente degli illustri precedenti che si incontrano proprio nella regione dove si sono incrociati i più diversi apporti delle ricerche dello stesso Antonello, di Mantegna e di Bellini, sensibili alla lezione di Piero della Francesca: il Veneto, importante tappa di Antonello tanto nella sua formazione quanto per le ripercussioni su tutto il panorama della pittura veneta.

Una prima intrigante prospettiva con punto di vista molto ribassato la troviamo in quel che è rimasto del ciclo padovano agli Eremitani: la Cappella Ovetari, commissionata nel 1448 a Nicolò Pizolo, Andrea Mantegna, Antonio Vivarini e Giovanni D’Alemagna, testo capitale nella storia dell'illusionismo prospettico. Vi lavorano dunque, accanto ai due alti rappresentanti di attardate maniere tardogotiche veneziane, i due giovani padovani gravitanti nell'orbita della bottega di Francesco Squarcione, discusso personaggio del panorama artistico della città. I due giovani pittori, che presto rileveranno anche alcuni degli episodi affidati ai più anziani maestri dopo che questi abbandoneranno il ciclo, si cimentano in visioni complesse e ardite prospettive ${ }^{\mathrm{I}}$.

Mantegna, presto riconosciuto come grande maestro di sapienza prospettica, scorcia vertiginosamente l'episodio di san Giacomo condotto al martirio: il punto di fuga, posto al di sotto del margine inferiore dell'affresco, offre un'inconsueta visione ribassata, annuncio del sott'in su nell'oculo mantovano della Camera Picta, capolavoro che apre, con forte anticipo sui tempi, il grande capitolo dell'illusionismo prospettico. È il passaggio a una concezione rinnovata della funzione della prospettiva, non più e non solo misura delle cose, ma strumento di fascinazione, mezzo per sollecitare una partecipazione emotiva oltre che intellettuale.

Che Antonello abbia visto le opere del padovano resta un'ipotesi; di certo è plausibile una conoscenza, magari solo indiretta, per il tramite di Giovanni Bellini, delle invenzioni del Mantegna ma, lo scorcio degli Eremitani, presenta più di una superficiale somiglianza d'impianto con il san Sebastiano di Dresda. La restituzione prospettica dell'affresco mostra alcune interessanti soluzioni sperimentate dal padovano ed evidenzia che,

I4. Alla morte di Giovanni d'Alemagna, Vivarini abbandona il lavoro e vi subentrano Bono da Ferrara e Ansuino da Forlì. Le vicende legate al ciclo pittorico sono piuttosto controverse e i dibattuti documenti relativi hanno dato luogo a divergenti attribuzioni, ora complicate dal drammatico stato di conservazione degli affreschi causato dal bombardamento del 1944. Gli episodi citati in questo lavoro sono però concordemente attribuiti al giovane Mantegna. 
anche qui, come nell'opera di Antonello, sotto un apparente rigore geometrico si nascondano alcune deroghe alla norma.

Ricostruendo l'impianto prospettico s'individua con estrema facilità il punto di vista, ma risulta evidente che non tutte le parallele convergono nel medesimo punto. Si possono, infatti, rintracciare ben tre punti di fuga verso i quali convergono tre gruppi di parallele (si tratta dunque di una complessa prospettiva accidentale) la cui particolare disposizione dichiara l'intenzionalità della scelta. L'architettura in primo piano, l'imponente arco sotto il quale si svolge l'episodio di san Giacomo condotto al martirio, si organizza intorno ad un punto di fuga esterno al quadro che va a definire la linea di orizzonte. Sulla linea d'imposta dell'affresco, identificabile come linea di terra, si collocano invece gli altri due punti di fuga, rispettivamente del gruppo di architetture poste a destra e degli edifici che s'intravedono al di là dell'arco; l'ipotetica linea di terra del primo gruppo viene così a coincidere con l'orizzonte dei due blocchi architettonici secondari; il punto di fuga del gruppo in lontananza coincide con il punto di distanza dell'arco. Anche in questo caso, come per il san Sebastiano, ci troviamo di fronte a deroghe troppo studiate perché siano considerate errori; anche qui le deformazioni prospettiche sembrano orientate a potenziare l'effetto drammatico sollecitando il coinvolgimento del soggetto osservante. La particolare interpretazione della prospettiva operata da Mantegna può rivelarsi ancora utile alla comprensione del san Sebastiano. Un'altra opera del Mantegna, ormai riconosciuta come qualche cosa di più di un puro virtuosismo, il Cristo in scorcio conservato alla Pinacoteca di Brera, può illuminare sulla particolare posizione del san Sebastiano e sulla fruizione da questo sollecitata.

\section{La fruizione richiesta}

Nella seconda metà del xv secolo, nell'ambito del Nord Italia, ebbe luogo un'importante evoluzione storico-artistica: a singole icone vennero aggiunte delle figure; la rappresentazione in forma di ritratto del personaggio sacro fu così narrativizzata e collegata ad uno specifico momento della sua vita e della sua sofferenza ... . Il dipinto del Mantegna può rientrare, dal punto di vista tematico, nel tipo di imago pietatis rappresentato dal Compianto del Bellini $[\ldots]^{15}$. 
Il Cristo del Mantegna si presenta però, per diverse ragioni, come imago pietatis decisamente atipica. In primo luogo la figura di Cristo non è rappresentata a mezzo busto ma intera, ciò nonostante - attraverso il taglio dell'immagine, il vistoso scorcio, la fuoriuscita dei piedi oltre il bordo del quadro - l'opera mantiene la stretta vicinanza con l'osservatore, vicinanza fondamentale per il coinvolgimento sollecitato dalle immagini devozionali. La soluzione compositiva adottata dal Mantegna, dunque, pur risolvendosi in una presentazione dell'intero corpo, mantiene quel carattere di forte presenza allo sguardo dell'osservatore che è caratteristica delle immagini a mezzo busto destinate ad una privata contemplazione, immagini patetiche e ravvicinate che sono in grado di sollecitare una modalità di fruizione del tutto peculiare:

Le caractère intime de l'icône à mi-corps en faisait un instrument particulièrement bien adapté au culte privé en ce qu'il permettait à l'individu de s'identifier pleinement au personnage représenté. Cette image "en gros plan» conférait à la méditation l'aspect d'une paisible conversation en tête à tête; elle jouissait d'une grande faveur parce qu'elle "rapprochait» le fidèle de la divinité [... l'artiste mettait l'accent sur l'aspect affectif et privilégiait l'expression au détriment de l'action ${ }^{16}$.

E dunque:

[...] l'attention se porte sur une attitude particulière du spectateur, attitude qui n'est ni une soif de savoir et de conseils, que l'on cherche à assouvir par la contemplation $d$ historiae, ni la vénération et l'adoration d'imagines, mais qui consiste en une profonde expérience affective. On considère que le but premier de l'image est de créer chez le spectateur un certain état psychologique $[\ldots]^{17}$.

È stato osservato come il taglio adottato da Mantegna, non contraddicendo un'impostazione di questo tipo, sia funzionale all'esibizione di tutte e cinque le piaghe del corpo di $\mathrm{Cristo}^{\mathrm{I}}$, esibizione che risponde proprio a un'esigenza devozionale e che sollecita quell'approccio empatico di cui parlava Ringbom ${ }^{19}$.

Pur con le debite differenze, dovute in particolare alla diversa destinazione d'uso, è possibile leggere la ravvicinata immagine del san Sebastiano di Antonello alla luce di un'esigenza non dissimile da quella ipotizzata per le immagini a mezzo busto e per il Cristo di Mantegna. Il corpo imponente dipinto da Antonello si presenta, infatti, in primissimo piano e subordina a sé tutta la composizione esibendo le frecce conficcate nel corpo; queste

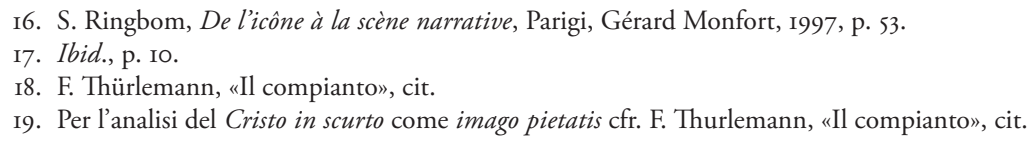


sono l'elemento più vicino all'osservatore, emergono in primo piano, rese plastiche e concrete dal proiettarsi delle ombre sul corpo: primo oggetto su cui far cadere l'attenzione, qualcosa di più di un semplice simbolo di martirio. La freccia più alta, quella che proietta l'ombra più corta, si trova sull'asse ideale che segna la porzione aurea della tavola, indizio che ne sottolinea l'importanza. Possiamo supporre che l'ostentazione delle frecce del martirio indirizzi ad un particolare tipo di ricezione dell'opera, sul genere di quella indicata per il Cristo del Mantegna, una ricezione dunque partecipata, in cui sia richiesto un coinvolgimento emotivo dell'osservatore, cosa che già ci aveva indicato il sovradimensionamento della figura rispetto alla coerenza prospettica.

L'organizzazione dello spazio indica la precisa posizione scelta dall'artista per il suo osservatore ideale. Pur ponendo ad una distanza notevole il punto di vista, Antonello realizza un'opera in cui, a livello percettivo, chi guarda si trova in una posizione estremamente ravvicinata rispetto al martire; le sue grandi dimensioni danno, infatti, la sensazione di poterlo quasi toccare, relegando invece in lontananza tutta l'ambientazione architettonica ${ }^{20}$. Ne risulta una posizione dell'osservatore decisamente contraddittoria, per la quale può venirci in aiuto quanto è stato osservato da Thürlemann per il Cristo del Mantegna:

[...] i piedi sono, relativamente allo spazio simulato, i più vicini all'osservatore. La testa invece, che permette all'uomo una vera comunicazione, che fa di lui una persona, è la più lontana dall'osservatore. Questa struttura contraddittoria, estrema vicinanza del corpo-lontananza della persona, accompagnata da un forte appello all'individuo, determina una modalizzazione contraddittoria del soggetto osservante: un'esortazione alla comunicazione, un dover comunicare, cui si accompagna simultaneamente l'impossibilità di una reale presa di contatto, un non poter comunicare. In altre parole, la comunicazione imposta è possibile a livello corporeo, come osservazione delle piaghe e dei segni della sofferenza; nello stesso tempo, però, non si attua né un vero dialogo umano, né una giustificazione nei confronti del sofferente ${ }^{21}$.

Non si vuole qui forzare un parallelo tra due opere così diverse per soggetto, per impostazione compositiva e per destinazione, si vuole solo sottolineare come la soluzione adottata da Antonello sia assolutamente non casuale ma comprensibile in un contesto artistico e devozionale che va compreso nel suo orizzonte di riferimento, va insomma guardato in relazione a quella che, possiamo supporre, fosse la modalità di fruizione di

2I. F. Thürlemann, «Il compianto», cit., p. 89. 
un'opera del genere sul finire del Quattrocento, quella fruizione empatica che l'interpretazione data per il Cristo in scurto può aiutarci a capire.

Detto questo va comunque osservato che può essere rintracciata una più stringente somiglianza tra le due opere. È stato notato come la figura di Cristo di Mantegna non fosse un'immagine prospettica, ma rispondesse bensì alle regole indicate per la rappresentazione della figura umana in proiezione ortogonale, infatti,

[...] occorre anzitutto sottolineare come, quasi a dispetto della sua fama quale saggio di straordinaria e ingegnosa "bravura", la struttura prospettica dell'opera presenti vistose incongruenze che impediscono di rintracciare un punto di vista unitario per tutta la composizione ${ }^{22}$.

Si nota, in effetti, che per rispondere ad una costruzione prospettica, i piedi avrebbero dovuto essere molto più grandi e il corpo subire una progressiva riduzione metrica culminante in una testa più piccola di quella dipinta da Mantegna. Una proiezione, dunque, più parallela che centrale e che rimanda a quanto illustrato nel codice Huygens in relazione alle modalità per la rappresentazione della figura umana in scorcio secondo proiezioni parallele ${ }^{23}$. L'invenzione del Mantegna

[...] si presenta piuttosto come il frutto di una sottile, raffinatissima mediazione, quasi un compromesso, tra le ragioni della verosimiglianza naturalistica e prospettica e quelle del rispetto dell'armonia e delle proporzioni del corpo umano [...]. Un compromesso di cui si colgono tuttora il segno e il fascino ambiguo $[\ldots]^{24}$.

Si è visto come il Sebastiano di Antonello non subisca riduzioni metriche relazionabili alla distanza dall'osservatore; la figura è inquadrata dal basso, si vede, infatti, la base del mento e non si legge con chiarezza il limite superiore delle spalle ma, per quanto riguarda le sue reali dimensioni, Sebastiano risulta visto attraverso una proiezione non perfettamente conica, che ricorda da vicino quanto raffigurato nel codice citato per il Mantegna. Quale che sia la fonte di Antonello, se pure ce ne fu una per la redazione del san Sebastiano, quello che si può per certo dire è che l'artista forza l'immagine a suo piacimento arrivando, anch'egli, a un compromesso tra le ragioni della scienza e quelle dell'espressione.

\footnotetext{
22. F. Frangi, Cristo morto, Milano, TEA editore, 1996, p. 43.

23. Non si sa se tali procedure fossero già in uso al tempo della realizzazione del Cristo, ma non si può escludere che Mantegna vi sia arrivato anche per deduzione.

24. F. Frangi, Cristo morto, op. cit., p. 53.
} 


\section{La struttura compositiva}

«[...] Capolavoro è il San Sebastiano di Dresda. Corpo ed ambiente si rispondono per forme analoghe» ${ }^{25}$. Con questa formula, Longhi ci ricorda il rigore geometrico come espressione della forma monumentale di Antonello, la capacità del messinese di tradurre in forma migliore ogni oggetto della percezione, la volontà di costruire le immagini su una struttura semplice ma solenne nei suoi rapporti interni. Si tratta, secondo Longhi, di una derivazione pierfrancescana, che però Antonello traduce in una più sensibile ed epidermica, più emotiva rappresentazione ${ }^{26}$.

Lo schema compositivo è semplice e rigoroso, la tavola ha i suoi lati in un rapporto perfetto di uno a due, due quadrati sovrapposti la cui linea di congiunzione, la metà della tavola, cade esattamente sul bordo superiore della loggia. La cornice più bassa della stessa loggia segna un'altra forte orizzontale che sfugge, a prima vista, ad una chiara collocazione geometrica ma si rivela, invece, giustificata: dividendo i due quadrati che formano la tavola e individuando così altri quadrati, in rapporto con i primi di uno a due, si rintraccia una misura ricorrente tanto da poter essere definita il modulo. È una misura in rapporto di uno a due con il lato corto e che corrisponde all'esatta lunghezza della mattonella come si presenta nel margine inferiore del dipinto; è una grandezza esattamente doppia rispetto allo spazio occupato dal frammento di colonna; una misura che giustifica anche la cornice della loggia trovandosi questa ad un modulo di distanza dalla linea d'orizzonte.

La figura del santo è ruotata e leggermente inclinata, mostra la sua parte destra ed emerge, per quasi la metà della sua lunghezza, sul cielo. Il corpo di Sebastiano taglia il balcone del loggiato in due parti, ne risultano due segmenti, uno a destra e uno a sinistra, che sono tra loro in un rapporto aureo ${ }^{27}$ come aureo è il rapporto tra l'apertura dell'arco e la sua altezza. Da una più attenta osservazione dei rapporti aurei s'individua un altro elemento modulare - è una misura assai ricorrente - che regola le proporzioni degli elementi architettonici: lo spazio occupato dalla colonna al margine destro del quadro, il cerchio che decora la mattonella e l'asse

\footnotetext{
25. R. Longhi, Breve ma veridica storia della pittura italiana, Milano, Rizzoli «B.u.r», I997, p. 88.

26. Non vi è però alcun documento che attesti una diretta conoscenza da parte di Antonello delle opere di Piero della Francesca. Per il problema dell'interpretazione longhiana della matrice geometrica del messinese e dei suoi rapporti con la pittura centro italiana si rimanda alle osservazioni di Mauro Lucco «Venezia», cit.

27. La sezione aurea, già definita dai matematici greci intorno al vi secolo a.c., indica uno specifico rapporto tra due grandezze per cui la maggiore è medio proporzionale tra la minore e la somma delle due. Il numero costante, detto $\phi$, e derivato dalla formula $(\mathrm{I}+\sqrt{5}) / 2$, equivale approssimativamente a $\mathrm{I}, 6 \mathrm{I} 8$.
} 
della testa del santo. Da questo segmento, in una progressione armonica, si danno le altre misure.

La composizione è geometricamente articolata, cristallina e quasi pierfrancescana ma l'immagine non si esaurisce nell'individuazione di una selva di rapporti aurei: la luce ammorbidisce i contorni stemperando il rigore formale e la rigida astrazione geometrica che governa gli oggetti e il corpo del martire mentre il colore sfuma i passaggi da piano a piano addolcendo le gradazioni di tono e facendosi, dunque, più naturale, lontano dalle perfette astrazioni del primo Quattrocento. Antonello tenta la sintesi tra le supreme armonie geometriche, una luce che ammorbidisce ma non smette di rilevare la forma e una dimensione più naturale, un rapporto tra umanità e natura.

\section{Una strana piazza}

Gran parte del fascino di quest'opera risiede nella strana e ambigua atmosfera della piazza. Piazza reale o invenzione di Antonello? Già Longhi aveva cercato di svelare il mistero dell'ambientazione andando a cercare precedenti e riferimenti pittorici e indicando una parte dell'organismo architettonico del Martirio di San Cristoforo agli Eremitani, un dettaglio interessante in questa rete di riferimenti mantegneschi di Antonello ${ }^{28}$. Oltre gli archi della tavola di Dresda sembra di intravedere dell'acqua, acqua che fa pensare alla laguna veneta e non va dimenticato, infatti, che se pure l'opera è stata dipinta a Messina, era comunque destinata ad una chiesa veneziana. I comignoli cilindrici, al di là del loro valore formale, sono un chiaro indizio, essendo tipicamente veneti, così come il balcone che corre sopra il porticato lo ritroviamo, oltre che nell'opera citata del Mantegna, anche nell'affresco del medesimo ciclo padovano dedicato al Battesimo di Ermogene; simili balconi, dai quali casuali osservatori assistono all'evento, appaiono già in opere trecentesche padovane, ce ne offre un'immagine quel grande narratore trecentesco che fu Altichiero nei Funerali dell'apostolo Giacomo nella Basilica di sant'Antonio.

Tornando alle indicazioni di Longhi e dando un altro sguardo al Martirio di San Cristoforo e al nostro San Sebastiano, possiamo vedere come in quest'ultimo, se pure in forma ridotta, appaia un accenno di quel pergolato che in Mantegna copre la piazza dell'evento; un piccolo

28. È da notare, inoltre, che il san Sebastiano era il laterale sinistro di un trittico il cui laterale destro era dedicato proprio a san Cristoforo. 


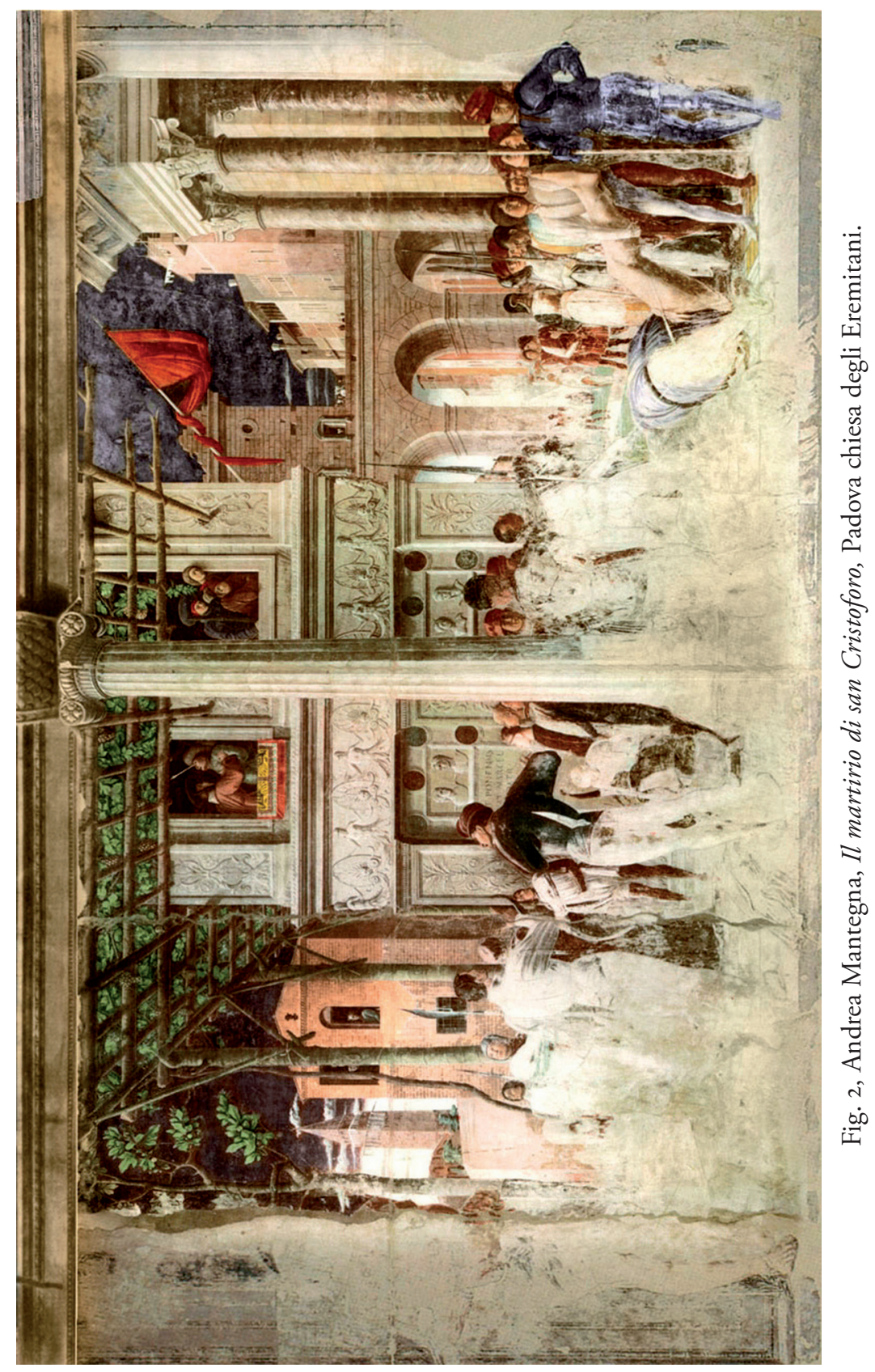


pergolato si vede, infatti, sul tetto dell'edificio a sinistra, quasi al limite della tavola di Antonello. Più di questo, è interessante ritrovare lo stesso personaggio nelle due opere: l'arciere che volta le spalle al san Sebastiano assomiglia, per la posizione e per l'abbigliamento, alla figura che, a sinistra in primo piano, guarda fuori scena nell'affresco di Mantegna, anch'esso dunque dando le spalle al santo e al suo martirio, anch'esso indifferente, poggiato su quella lancia che immaginiamo usata per ferire il santo. Sembra quasi che Antonello abbia dipinto il suo Sebastiano immaginandolo nella piazza che, nell'affresco padovano, si intravede oltre gli archi. Quegli edifici sulla destra dipinti da Mantegna ricordano le case a sinistra della tavola, le stesse aperture ad arco, lo stesso balconcino, forse non è solo una casualità (si veda fig. 2).

\section{Per concludere: il corpo del santo}

Sebastiano domina la scena, alto e imponente, statuario e solido come una colonna, come una perfetta forma volumetrica, è stato messo in relazione, da molti autori, al frammento di colonna che gli sta accanto. Con le braccia legate dietro la schiena, lo sguardo rivolto verso l'alto, si presenta leggermente ruotato in una posizione che, negando la rigida frontalità ancora preferita da molti pittori quattrocenteschi nella rappresentazione delle figure sacre, stabilisce un più vivo contatto con quanto sta fuori del quadro.

Le ricerche anatomiche saranno oggetto di studio della generazione successiva, iniziate da Leonardo verranno approfondite nel nuovo secolo, ma l'attenzione al corpo umano, alla sua potenzialità espressiva, è già una conquista dell'Umanesimo. Antonello dipinge una figura credibile e approfitta della nudità suggerita dalla diffusa interpretazione iconografica del racconto sacro del martirio per indugiare nella definizione del corpo: sottolinea i muscoli delle gambe, il modellato del ventre, le ombre del collo. Un apparente naturalismo è quanto ne risulta, ma si è visto come questa tavola nasconda sottili ambiguità e quello che appare, dunque, potrebbe nascondere altro. La naturalezza della posa, con quella leggera torsione del corpo, che fa apparire Sebastiano più vero e spontaneo di tante altre figure dipinte negli stessi anni, nasconde qualche piccola forzatura: il braccio destro è troppo ruotato e mostra al nostro sguardo la parte posteriore del gomito, quasi fosse disarticolato oltre che troppo lungo; il bicipite si trova un poco sovrapposto alla zona dei pettorali come fosse esteso in avanti anziché indietro. Troppo lungo è in realtà tutto il torace, 
leggermente sproporzionato rispetto alla parte inferiore del corpo nonostante la riduzione che, in una rappresentazione in scorcio, dovrebbe subire.

La naturalezza risulta qui frutto di piccole alterazioni anatomiche, soluzioni non sempre probabili, a ribadire, forse, che quanto stiamo vedendo, che quanto ci appare reale e concreto, che quanto ci sembra frutto di una capacità descrittiva che già tende a soluzioni di un naturalismo più spinto, è pur sempre un'apparizione che seduce per la sua morbidezza cromatica, per l'intrigante diffondersi delle ombre, per la razionale spazialità, per la presenza di carne ma che rivendica, però, il suo essere di un'altra natura, cosa lontana dalla concretezza del reale: l'essere spirituale del santo, l'essere opera d'arte della tavola.

L'impianto formale, l'espressione sensibile, si identifica con un particolare contenuto spirituale; il valore espressivo di ogni elemento deviante attesta la libertà di creazione e dimostra come qui la scienza sia strumento per una rappresentazione che non si esaurisce in una dimostrazione di bravura ma rivendica all'arte la sua autonomia nel disporre dei mezzi in funzione di un significato. 